[13] R. L. Mattson, J. Gecsei, D. R. Slutz, and I. L. Traiger, "Evaluation techniques for storage hierarchies," IBM Syst. J., vol. 9, pp. 78-117, 1970.

[14] J. B. Morris, "Demand paging through the use of working sets on the MANIAC II," Commun. Ass. Comput. Mach., vol. 15, pp. 867-872, Oct. 1972.

[15] J.-F. Pâris, personal communication, Feb. 1981.

[16] B. G. Prieve, "A page partition replacement algorithm," $\mathrm{Ph} . \mathrm{D}$. dissertation, Univ. California, Berkeley, 1974.

[17] J. Rodriguez-Rosell and J. P. Dupuy, "The design, implementation, and evaluation of a working set dispatcher," Commun. Ass. Comput. Mach., vol. 16, pp. 247-253, Apr. 1973.

[18] A. J. Smith, "A modified working set paging algorithm," IEEE Trans. Comput., vol. C-25, pp. 907-914, Sept. 1976.

[19] A. J. Smith, "Two simple methods for the efficient analysis of memory address trace data," IEEE Trans. Software Eng., vol. SE-3, pp. 94-101, Jan. 1977.

[20] J. R. Spirn, "Program locality and dynamic memory management," Ph.D. dissertation, Princeton Univ., Mar. 1973.
Domenico Ferrari (M'67), for a photograph and biography, see p. 79 of the January 1983 issue of this TRANSACTIONS

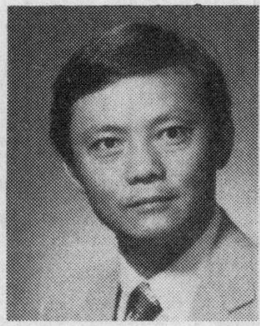

Yiu-Yo Yih received the B.E. degree in computer engineering from the University of Michigan, Ann Arbor, and the M.S. degree in computer science from the University of California, Berkeley.

He joined Bell Laboratories, Holmdel, NJ, as a member of the technical staff in May 1980. His interests include operating systems and pattern recognition. He is currently working towards a Ph.D. degree in computer science at Rutgers University.

\title{
The Study of a New Perfect Hash Scheme
}

\author{
M. W. DU, MEMBER, IEEE, T. M. HSIEH, K. F. JEA, AND D. W. SHIEH
}

\begin{abstract}
A new approach is proposed for the design of perfect hash functions. The algorithms developed can be effectively applied to key sets of large size. The basic ideas employed in the construction are rehash and segmentation. Analytic results are given which are applicable when problem sizes are small. Extensive experiments have been performed to test the approach for problems of larger size.
\end{abstract}

Index Terms-Hashing, perfect hash functions, rehash, segmentation.

\section{INTRODUCTION}

$\mathrm{H}$ ASHING has been considered as an effective means to organize and retrieve data in program design and has been widely used in database management, compiler construction, and many other applications. In order to use hashing techniques in a specific application, one has to first choose a suitable hash function, then select a method for collision resolution. Quite a number of ways have been proposed to design hash functions [2], [13], [16], [17], [21]. Two collision resolving methods, chaining and open addressing, have also been explored in many papers [12], [15] [17]-[20], [23].

If a hash function can be found which is one-to-one from the set of keys in the key space to the address space then that

Manuscript received April 20, 1981; revised December 27, 1982. This work was supported in part by the National Science Council of R.O.C. under Grant NSC-68E-0201-04(05). Part of this paper was presented at COMPSAC'80, Chicago, IL, October 1980.

M. W. Du is with the Institute of Computer Engineering, National Chiao Tung University, Hsinchu, Taiwan, R.O.C.

T. M. Hsieh is with the Institute of Electronic Engineering, National Chiao Tung University, Hsinchu, Taiwan, R.O.C. and the Department of Electronic Engineering, Chung Yuan University, Chung Li, Taiwan, R.O.C.

K. F. Jea is with the Department of Computer Science, University of Wisconsin, Madison, WI 53706.

D. W. Shieh is with the System Development Center, Institute for Information Industry, Taiwan, R.O.C. hash function will become much easier to use since the bothersome key collision problem can be avoided. There exist a number of methods for the design of a one-to-one hash function [1], [3], [8]-[10], [22] or called perfect hashing function in [22]. In suitable situations their methods may yield good hash functions as far as the memory space used or the execution time are concerned.

In this paper an entirely new approach is proposed for the design of perfect hash functions. An indicator table is used in the construction of a perfect hash function with table size in linear proportion to the number of keys. Compared to other methods proposed in the literature for designing perfect hash functions [1], [3], [8]-[10], [22], the construction procedures here have the advantages that they are easy to implement and can be effectively applied to key sets of large size.

The basic ideas employed in our construction are rehash and segmentation. In Sections II-IV we will show how random hash functions are organized by using a hash indicator table to construct a desired perfect hash function. Analytic results are given which are applicable when problems sizes are small. In Section V, two algorithms are designed for the construction of the hash indicator table. Extensive experiments have been performed to test the new approach for problems of large size. The results are presented in Section VI.

Formulas for calculating probabilities and expectations discussed in the context are listed in the Appendix.

\section{Random Hash Functions}

We shall consider random hash function first. Fig. 1 shows the basic model which will be considered throughout this paper. In the model, a set of $n$ nonequal keys $K_{1}, K_{2}, \cdots, K_{n}$ in the key space is mapped into an address space with $m$ entries 




Fig. 1. Basic hash model.

by a hash function $h$. No interrelations will be assumed to exist among the $n$ keys. The keys can be thought of as just arbitrary binary strings. Therefore, the hash function can be fully characterized by the listing of the values $h\left(K_{i}\right)$ for all $1 \leqslant i \leqslant n$, where $1 \leqslant h\left(K_{i}\right) \leqslant m$. $h$ is called a random hash function whenever all the $h\left(K_{i}\right)$ 's are selected randomly from $\{1,2, \cdots, m\}$.

We say that a collision occurs if for some $i \neq j, h\left(K_{i}\right)=h\left(K_{j}\right)$. In such situation, we also say that $K_{i}$ and $K_{j}$ are collided keys under $h$.

Definition 1: $h$ is a perfect hash function iff no collision occurs in $h$.

We define below a probability which will be used to measure the likelihood of constructing perfect hash functions.

Definition 2: Let $F$ be a set of possible distinct functions obtainable from a construction procedure $P$, with the functions mapping from a set of keys into an address space. Assume that there are $n_{p}$ perfect hash functions among the $n_{F}$ functions in $F$. If a hash function $h$ is selected randomly from $F$, the probability of $h$ being perfect, denoted as $\operatorname{pbp}(h)$, is equal to $n_{p} / n_{F}$.

It should be noted that $h$ is a formal name representing a function selected randomly from $F$ in Definition 2 . It can also be thought of as the formal name representing a function constructed by the procedure $P$. Clearly, $\operatorname{pbp}(h)$ depends entirely on how procedure $P$ constructs perfect hash functions.

As an example, assume that $h$ is a mapping function selected randomly from $F_{n \times m}$, the set of all functions that map $n$ keys into an address space with $m$ entries. The chances that $h$ is a perfect hash function are ordinarily quite small. Actually, $\operatorname{pbp}(h)=0$ if $n>m$, and is equal to $m ! /(m-n) ! \times\left(1 / m^{n}\right)$ if $n \leqslant m$. When $n=m=10, \operatorname{pbp}(h)=0.0003629$. Even when $m$ is much larger than $n$, the probability is still very small. When $n=23$ and $m=365$, we have the famous "birthday paradox" [7], [13]. The probability is only 0.4927 , still less than half!

We say that a hash function $h$ has $k$ singletons if there are $k$ entries in the address space with a single key hashed by $h$ to each of them. In general, we can find the probability for $h$ having exactly $k$ singletons by the following theorem.

Theorem 1: Assume that $h$ is a random hash function from $n$ keys to an address space of size $m$. Let us denote the probability distribution of the number of singletons $k(0 \leqslant k \leqslant$ $\min (m, n))$ as $P_{k}(n, m)$. Then

$$
P_{k}(n, m)=\frac{e_{k}(n, m)}{m^{n}}
$$

where

$$
e_{k}(n, m)=n !\left(\begin{array}{c}
m \\
k
\end{array}\right) \sum_{r=0}^{n-k}(-1)^{r}\left(\begin{array}{c}
m-k \\
r
\end{array}\right) \frac{(m-r-k)^{n-r-k}}{(n-r-k) !} .
$$

This theorem can be proved by first finding the number of possible $h$ functions having at least $j$ singletons, using the ordinary enumerator $\left(x_{1}+x_{2}+\cdots+x_{m}\right)^{n}$ of the number of ways in which $n$ distinct objects can be distributed into $m$ distinct cells, then applying the principle of inclusion and exclusion [6], [14] to get $p_{k}(n, m)$.

By using the distribution function in Theorem 1, closed form for the mean of $P_{k}(n, m)$ can be found [4] , [11] as

$$
E[k]=n \times\left(1-\frac{1}{m}\right)^{n-1} \text {. }
$$

Let us assume that $n=\alpha m$, the formula $n \times(1-(1 / m))^{n-1}$ approaches $n \times e^{-\alpha}$ when $n$ becomes very large. Therefore, is $h$ is selected randomly from $F_{n \times m}$, ordinarily it will be far from being a perfect hash function. To improve this situation, some information about the keys should be used to construct the hash function. In the next section we use rehash to construct a new hash function from a number of hash functions selected from $F_{n \times m}$. Information related to the keys and the hash functions selected is kept in a table called hash indicator table (HIT).

\section{First Level Rehash}

Fig. 2 shows the first-level rehash model where the hash function $h$ is constructed from a number of hash functions, $h_{1}, h_{2}, \cdots, h_{s}$, selected randomly from $F_{n \times m}$. In the figure, the HIT has the same number of entries as that of the address space. In fact, entry $d$ in HIT corresponds to entry $d$ in the address space.

The contents in HIT can be defined by the following Pidgin Algol program.

\section{Procedure 1 [Procedure to Construct First-Level HIT] :}

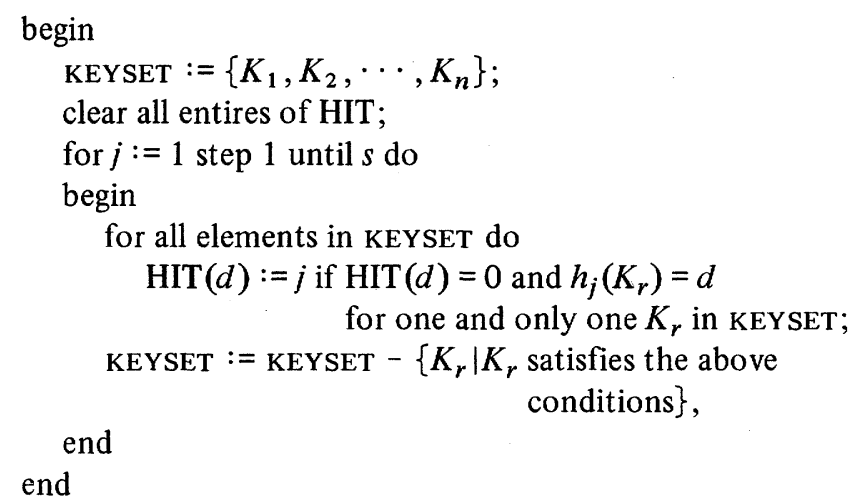

The first-level composite hash function $h$ can be defined as follows:

$$
\begin{array}{rlrl}
h(K)=h_{i}(K)=d & & \text { if } \operatorname{HIT}\left(h_{r}(K)\right) \neq r \text { for } r<i \\
& & \text { and } \operatorname{HIT}\left(h_{i}(K)\right)=i, \\
& =\text { undefined } & & \text { otherwise. }
\end{array}
$$

It can be seen that if $q$ hash functions are selected for composing $h$, a table HIT of width $\left[\log _{2}(q+1)\right]$ bits is required. 


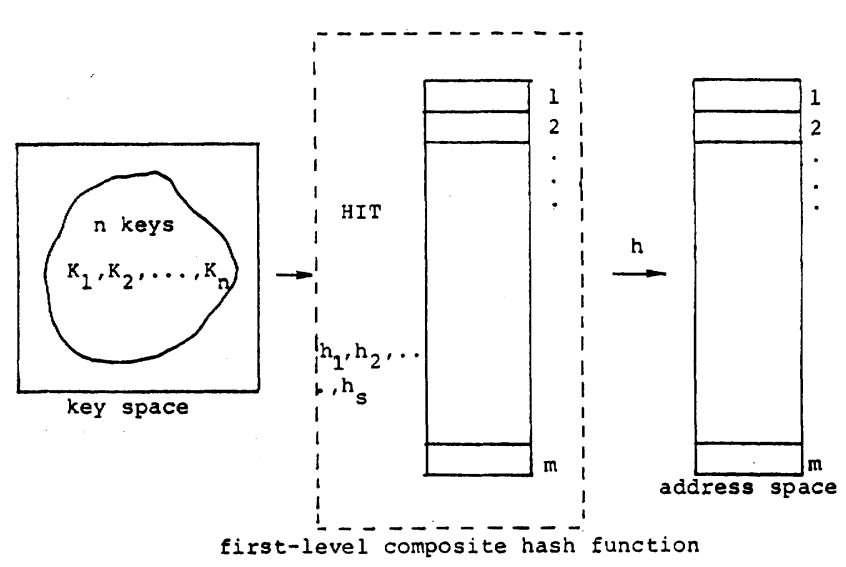

Fig. 2. First-level rehash model.

TABLE I

Hash Functions $h_{1}, h_{2}, h_{3}$ FOR ExAMPLE 1

\begin{tabular}{|c|c|c|c|c|c|c|c|c|}
\hline $\begin{array}{l}\text { hash } \\
\text { function }\end{array}$ & $k_{2}$ & $k_{2}$ & $k_{3}$ & $k_{4}$ & $k_{5}$ & $k_{6}$ & $k_{7}$ & $k_{8}$ \\
\hline$h_{1}$ & 4 & 5 & 4 & 6 & 2 & 6 & 2 & 9 \\
\hline$h_{2}$ & 5 & 8 & 9 & 2 & 4 & 4 & 9 & 2 \\
\hline$h_{3}$ & 7 & 6 & 5 & 5 & 2 & 4 & 9 & 8 \\
\hline
\end{tabular}



Fig. 3. HIT constructed by Procedure 1 of Example 1.

To find $h(K)$ for a key $K$ in the key space, we apply the hash functions $h_{1}, h_{2}, \cdots, h_{s}$ on $K$ in turn until $\operatorname{HIT}\left(h_{i}(K)\right)=i$. If the search fails, $h(K)$ is undefined.

Example 1: Assume that the key set is $\left\{K_{1}, K_{2}, \cdots, K_{8}\right\}$, and that the address space is from 1 to 9 . The hash functions selected are $h_{1}, h_{2}, h_{3}$, as defined by Table I. The circles in each row $h_{j}$ in the table indicate where the $h_{j}$ contributes singletons.

The HIT constructed by Procedure 1 is given in Fig. 3. We can see that $K_{2}$ and $K_{8}$ find their right places by $h_{1} . K_{4}$ by $h_{2}, K_{1}$ and $K_{6}$ by $h_{3}$. Note that $h_{2}\left(K_{1}\right)=5$ is a singleton in the second row of $h_{2}$, however, since 5 has been already occupied by $K_{2}$ through the mapping of $h_{1}, K_{1}$ does not find its right position by $h_{2}$. The mapping of the composed function $h$ is undefined on the remaining keys $K_{3}, K_{5}$, and $K_{7}$.

Let us see how $h\left(K_{6}\right)$ can be calculated. When $h_{1}, h_{2}, h_{3}$ are applied on $K_{6}$ in turn, we have $\operatorname{HIT}\left(h_{1}\left(K_{6}\right)\right)=\operatorname{HIT}(6)=$ $(00)_{2}=0 \neq 1, \operatorname{HIT}\left(h_{2}\left(K_{6}\right)\right)=\operatorname{HIT}(4)=(11)_{2}=3 \neq 2$, HIT $\left(h_{3}\left(K_{6}\right)\right)=\operatorname{HIT}(4)=(11)_{2}=3=3$. Therefore, $h\left(K_{6}\right)=4$.

Suppose that $k$ keys have been hashed successfully into the address space after $s$ hash functions of $h_{j}$ 's are applied in the way described above. Given any one key $K$ of these $k$ keys, we can apply the $h_{1}, h_{2}, \cdots, h_{s}$ successively, consulting the HIT table, to find $h(K)$. We define $N E 1(n, m, s, k)$ as the

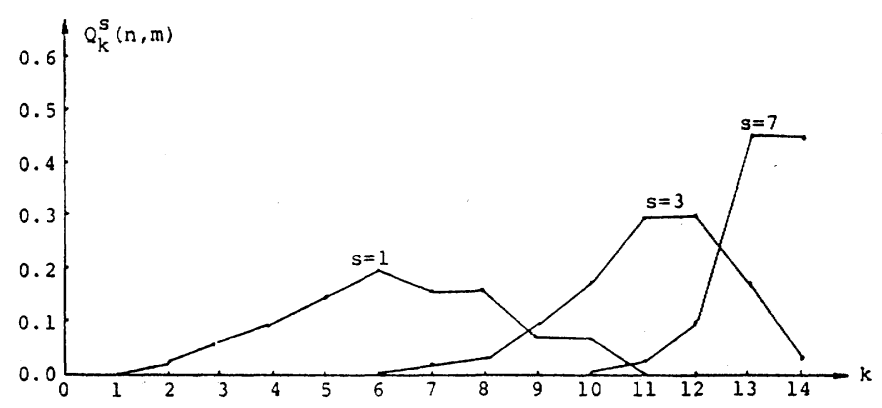

Fig. 4. Distribution diagram of $Q_{k}^{S}(n, m)$ with $n=14, m=17$, and $s=1,3,7$.

expected number of times it is required to apply $h_{i}$ 's for calculating $h(K)$ with $K$ among these $k$ keys.

Let $Q_{k}^{s}(n, m)$ be the probability of getting $k$ singletons by applying $h_{1}, h_{2}, \cdots, h_{s}$ in the way described previously, where each $h_{i}$ maps from the $n$ given keys to the address space of size $m$. Formulas for calculating $N E 1$ and $Q_{k}^{S}$ are given in the Appendix.

Example 2: The $Q_{k}^{s}(n, m)$ values for $n=14, m=17, s=$ $1,3,7$, are calculated and plotted in Fig. 4.

It can be seen that $Q_{k}^{1}(n, m)$ is exactly the probability distribution of the number of singletons of a random hash function and is equal to the distribution function $P_{k}(n, m)$ in (1).

By comparing the values of $Q_{k}^{1}(14,17)$ with those of $Q_{k}^{7}(14$, 17 ), one can observe that he may find more singletons in a first-level composite hash function than in a random hash function. The expectation of $k$ for $Q_{k}^{1}(14,17)$ is 6.673471 while that for $Q_{k}^{7}(14,17)$ is 13.280872 .

When $n$ singletons have been obtained by the above construction, we get a perfect hash function. The expectation of the number of times of applying $h_{i}$ 's in calculating $h(K)$ is $N E 1(14,17, s, 14)$. They are $1,1.579916,2.163818$ with $s=1,3,7$, respectively.

The probability of being perfect pbp for the first-level hash function composed from seven random hash function is $Q_{14}^{7}(14,17)$. It is much larger than $Q_{14}^{1}(14,17)$, the pbp of a random hash function.

When more and more random hash functions are selected to compose a first-level composite hash function, the probability of being perfect of the composite function will be certainly increased, but it will be increased very slowly. The reason is that the more singletons we already have, the fewer chances are there for the remaining keys to be hashed on unused entries in the address space. It has been found that dividing the address space into segments can be a more effective means to increase the pbp than using indefinitely many random hash functions, as will be described in the next section.

\section{SECond Level Rehash}

Fig. 5 shows the second-level rehash scheme.

The address space is divided into $q$ segments. Corresponding to segment $A_{i}$, which is of size $m_{i}$, we have a first-level composite hash function $h^{i}$ which maps $\left\{K_{1}, K_{2}, \cdots, K_{n}\right\}$ into $\left\{1, \cdots, m_{i}\right\} . \mathrm{HIT}_{i}$ is the hash indicator table of $h^{i}$ for indicating which $h_{j}^{i}$ is applied to get the value of $h^{i}$. $\operatorname{HIT}_{1}, \cdots, \mathrm{HIT}_{q}$ compose the hash indicator table HIT of the second-level composite hash function $H$.

The second-level composite hash function $H$ is defined as 


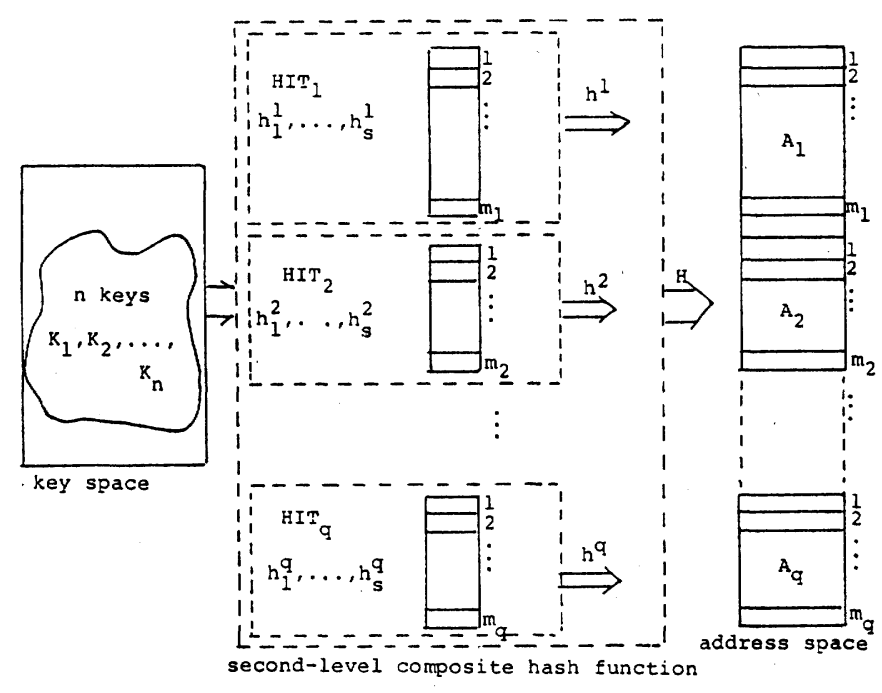

Fig. 5. Second-level rehash.

follows:

$$
\begin{aligned}
& H(K)=h_{j}^{i}(K)+\sum_{t=0}^{i-1} m_{t} \quad \begin{array}{l}
\text { if } i, j \text { can be found such that } \\
\operatorname{HIT}_{i}\left(h_{j}^{i}(K)\right)=j, \text { and }
\end{array} \\
& \begin{array}{l}
\operatorname{HIT}_{i}\left(h_{j}^{i}(K)\right)=j, \text { and } \\
\operatorname{HIT}_{i}\left(h_{r}^{i}(K)\right) \neq r \text { for } r<j, \text { and }
\end{array} \\
& \operatorname{HIT}_{a}\left(h_{b}^{a}(K)\right) \neq b \text { for } a<i \text {, } \\
& 1 \leqslant b \leqslant s \text {, } \\
& =\text { undefined otherwise. }
\end{aligned}
$$

That is, if a key $K$ can not find its right position in the first segment by $h^{1}$, it will try the second segment by $h^{2}$, and so forth.

The following program is an implementation of the hash function $H$.

\section{Procedure 2 [Procedure for Computing $H(K)$ ] :}

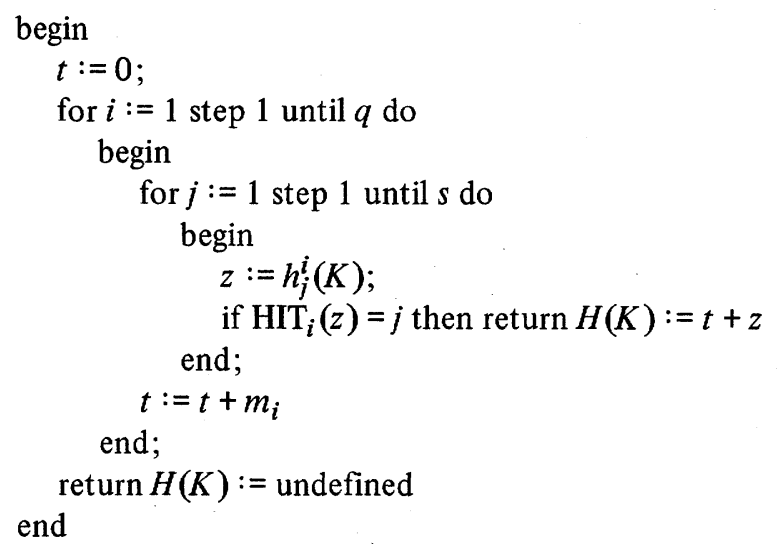

We use $R_{k}^{s}(n, \bar{m}, q)$ to denote the probability distribution of getting $k$ singletons by the second-level composite hash function $H$. The vector $\bar{m}$ is the partition on the address space. It can also be represented as $\left(m_{1}, m_{2}, \cdots, m_{q}\right)$. We shall also represent $\left(m_{1}, m_{2}, \cdots, m_{r}\right)$ as $\overline{r m} . \overline{q m}=\bar{m}$ by our convention.

Formulas for calculating $R_{k}^{s}(n, \bar{m}, q)$ are also given in the Appendix.

Example 3: To make things comparable to the results shown in Fig. 4, we let $n=14, m=17, \bar{m}=(13,4), s=1,3,7$, and get three second-level composite hash functions which map 14 keys into two segments of size 13 and 4 each. The $R_{k}^{s}(n, \bar{m}, q)$ values are calculated and plotted on Fig. 6 .

Let us examine the $R_{k}^{7}(14,(13,4), 2)$ case. The probability

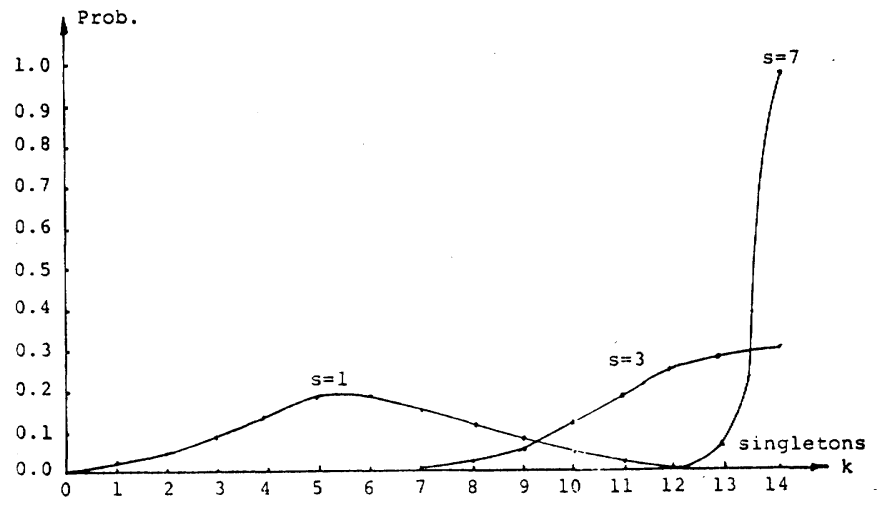

Fig. 6. Distribution diagram of $R_{k}^{S}(n, \bar{m}, q)$ with $n=14, \bar{m}=(13,4)$, $s=1,3,7$.

for the second-level hash function $H$ to be perfect is $R_{14}^{7}(14$, $(13,4), 2)$, which is greater than 97.8 percent!

Like in the first-level analysis, suppose that $k$ keys have been hashed successfully into the address space after the construction of the second-level hash function. Given any one key $K$ of these $k$ keys, we can apply the $h_{1}^{1}, h_{2}^{1}, \cdots, h_{s}^{1}, h_{1}^{2}, h_{2}^{2}, \cdots$, $h_{s}^{2}, \cdots$ successively, consulting the second-level HIT table, to find $H(K)$. Similarly, we define $N E 2(n, \bar{m}, q, s, k)$ as the expected number of times it is required to apply $h_{j}^{i}$,s for calculating $H(K)$ with $K$ among these $k$ keys. Formulas for calculating $N E 2$ are given in the Appendix.

Example 3 (Continued): $N E 2(n, \bar{m}, q, s, n)$ is the expected number of times to apply the $h_{j}^{i}$, s functions to calculate $H(K)$ for any key $K$, if the second-level composite hash function is perfect. Using (A9) in the Appendix to calculate NE2(14, $(13,4), 2, s, 14)$, we get $1.275607,2.286545,3.273526$ for $s=1,3,7$.

\section{Construction of the Hash Indicator table}

According to the way the hash indicator table is defined in Section III, a multipass procedure can be designed for the construction of the HIT. Let thet set of keys be $\left\{K_{1}, K_{2}, \cdots\right.$, $\left.K_{n}\right\}$. Assume that the partition of the address space $\left(A_{1}\right.$, $\left.A_{2}, \cdots, A_{q}\right)$ of size $\left(m_{1}, m_{2}, \cdots, m_{q}\right)$ and the hash functions $h_{1}^{1}, \cdots, h_{s}^{1}, h_{1}^{2}, \cdots, h_{s}^{2}, \cdots, h_{1}^{q}, \cdots, h_{s}^{q}$ are all given. The following is a HIT construction procedure.

Procedure 3 (Static Procedure to Construct HIT's):

begin

KEYSET : $=\left\{K_{1}, K_{2}, \cdots, K_{n}\right\}$;

clear all entries of $\mathrm{HIT}_{i}$;

for $i:=1$ step 1 until $q$ do

for $j:=1$ step 1 until $s$ do

begin

for all elements in KEYSET do

$$
\begin{gathered}
\operatorname{HIT}_{i}(d):=j \text { if } \operatorname{HIT}_{i}(d)=0 \text { and } h_{j}^{i}\left(K_{r}\right)=d \\
\text { for one and only one } K_{r} \\
\text { in KEYSET; } \\
\text { KEYSET }:=\text { KEYSET }-\left\{K_{r} \mid K_{r}\right. \text { satisfies the above } \\
\text { conditions }\} ;
\end{gathered}
$$

end;

if KEYSET = empty then HIT of a perfect hash function has been assigned else it fails to find a perfect hash function end 
TABLE II

Hash Functions for Example 4

\begin{tabular}{|c|c|c|c|c|c|c|c|c|c|}
\hline $\begin{array}{c}\text { hash } \\
\text { function }\end{array}$ & $\mathrm{k}_{1}$ & $\mathrm{~K}_{2}$ & $\mathrm{k}_{3}$ & $\mathrm{~K}_{4}$ & $\mathrm{k}_{5}$ & $\mathrm{k}_{6}$ & $\mathrm{k}_{7}$ & $\mathrm{~K}_{8}$ & $\mathrm{~K}_{9}$ \\
\hline $\mathrm{h}_{1}^{1}$ & 4 & 5 & 4 & 6 & 2 & 6 & 2 & 9 & $(1)$ \\
\hline $\mathrm{h}_{2}^{1}$ & 5 & 8 & 9 & 2 & 4 & 4 & 1 & 2 & 8 \\
\hline $\mathrm{h}_{3}^{1}$ & $(7)$ & 6 & 1 & 5 & 2 & 4 & 9 & 8 & 5 \\
\hline \hline $\mathrm{h}_{1}^{2}$ & 1 & 3 & 2 & 4 & 2 & 3 & 2 & 5 & 3 \\
\hline $\mathrm{h}_{2}^{2}$ & 2 & 4 & 5 & 2 & 5 & 5 & 3 & 1 & 3 \\
\hline $\mathrm{h}_{3}^{2}$ & 5 & 2 & $(1)$ & 1 & 5 & 4 & 1 & 3 & 2 \\
\hline
\end{tabular}


Fig. 7. HIT constructed by Procedure 3 of Example 4 .

Example 4: Assume that nine keys $K_{1}, K_{2}, \cdots, K_{9}$ are to be hashed perfectly to an address space of size 14 . The address space is partitioned into $\left(A_{1}, A_{2}\right)$ of size $(9,5)$. The hash functions used are $\left\{h_{1}^{1}, h_{2}^{1}, h_{3}^{1}\right\},\left\{h_{1}^{2}, h_{2}^{2}, h_{3}^{2}\right\}$ as defined by Table II. Again, as in Example 1, the circles in each row $h_{j}^{i}$ in the table indicate where the $h_{j}^{i}$ contributes singletons.

Fig. 7 shows the HIT constructed by Procedure 3 .

Procedure 3 works on an address space with the segments preset to $\left(m_{1}, \cdots, m_{q}\right)$. In contrast to this, the size of the address space and the segments can be set in a dynamic manner, as the following procedure suggests.

Procedure 4 [Dynamic Procedure to Construct HIT (or H)] : ; assume that the size limitation of the address space is $m$. begin

KEYSET : $=\left\{K_{1}, \cdots, K_{n}\right\}$;

for $i:=1$ step 1 until a large number $M$ do begin

let $m_{i}$ be the size of KEYSET;

$m:=m-m_{i}$;

if $m<0$, then it fails to find a perfect hash function, return;

reserve a table HIT $_{i}$ of length $m_{i}$ and clear it;

generate $s$ hash functions $h_{1}^{i}, \cdots, h_{s}^{i}$ that map from KEYSET into $\left\{1,2, \cdots, m_{i}\right\}$;

for $j:=1$ step 1 until $s$ do

begin

for all elements in KEYSET do

$$
\begin{aligned}
\operatorname{HIT}_{i}(d):= & j \text { if } \operatorname{HIT}_{i}(d)=0 \text { and } h_{j}^{i}\left(K_{r}\right)=d \\
& \quad \text { for one and only one } K_{r} \text { in } \\
& \text { KEYSET; }
\end{aligned}
$$

KEYSET := KEYSET $-\left\{K_{r} \mid K_{r}\right.$ which satisfies the above conditions $\}$;

if KEYSET = empty then HIT's of a perfect hash function has been assigned, return end

end

end

The expectation of the length of the HIT (or the length of the address space) and the expectation of the number of times it is required to apply $h_{j}^{i}$,s to find $H(K)$ for $K=$ any key $K_{j}$, with the HIT constructed by Procedure 4 , are defined as $L(n, s)$ and $\operatorname{NED}(n, s)$ respectively. They can be calculated by formulas in the Appendix.

Example 5: Let $s=1, n=3$. The probabilities of all possible cases constructed by Procedure 4 are depicted by the following probability tree:




The path from the root to each terminal node (indicated by an asterisk) represents a possible configuration $\left(l_{1}, \cdots, l_{q}\right)$, with $l_{1}+l_{2}+\cdots+l_{q}=3$. For example, the path from the root to $* A$ in the figure represents the configuration $(1,2)$. The probability to get $l_{1}=1, l_{2}=2$ is $Q_{1}^{1}(3,3) \times Q_{2}^{1}(2,2)$ the product of the $Q_{l_{i}}^{1}$ on the path from the root to the terminal node.

There are $3^{3}=27$ distinct functions which map three keys into $\{1,2,3\}$. Six of these contain 3 singletons, 18 of these contain 1 singletons, and 3 of these contain 0 singletons. Therefore, $Q_{3}^{1}(3,3)=6 / 27=2 / 9, Q_{2}^{1}(3,3)=0, Q_{1}^{1}(3,3)=$ $18 / 27=2 / 3, Q_{0}^{1}(3,3)=3 / 27=1 / 9$. Similarly, $Q_{2}^{1}(2,2)=1 / 2$, $Q_{1}^{1}(2,2)=0, Q_{0}^{1}(2,2)=1 / 2$. The probability tree can be simplified to the following tree by noting that $Q_{2}^{1}(3,3)=0$ and $Q_{1}^{1}(2,2)=0$ :

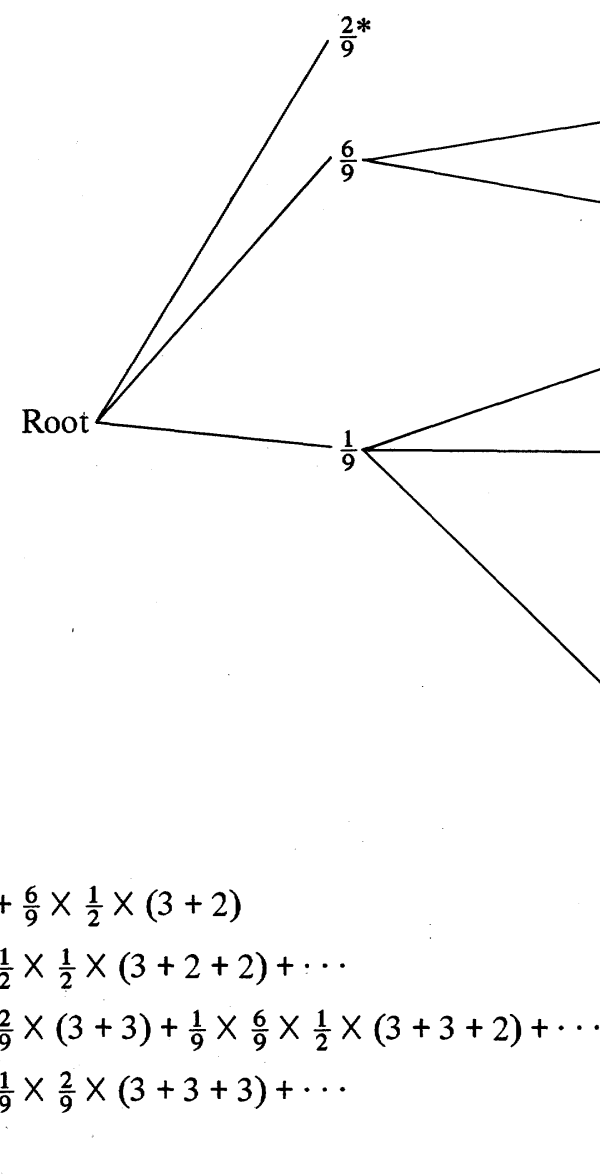

Similarly, by (A8), we have

$$
\begin{aligned}
N E D(3,1)= & \frac{2}{9} \times \frac{3}{3}+\frac{6}{9} \times \frac{1}{2} \times\left[\frac{1}{3} \times(1+2 \times 2)\right]+\cdots \\
& +\frac{1}{9} \times \frac{2}{9} \times\left[1+\frac{3}{3}\right] \\
& +\frac{1}{9} \times \frac{6}{9} \times \frac{1}{2} \times\left[1+\frac{1}{3} \times(1+2 \times 2)\right]+\cdots \\
& +\frac{1}{9} \times \frac{1}{9} \times \frac{2}{9} \times\left[1+1+\frac{3}{3}\right]+\cdots \\
= & 2 \frac{1}{8} .
\end{aligned}
$$

\section{EXPERIMENTAL RESULTS}

The formulas derived in the previous sections can be used to find important measures to characterize the construction of second-level perfect hash functions. However, they can be

By (A6) we have

$$
\begin{aligned}
L(3,1)= & \frac{2}{9} \times 3+\frac{6}{9} \times \frac{1}{2} \times(3+2) \\
& +\frac{6}{9} \times \frac{1}{2} \times \frac{1}{2} \times(3+2+2)+\cdots \\
& +\frac{1}{9} \times \frac{2}{9} \times(3+3)+\frac{1}{9} \times \frac{6}{9} \times \frac{1}{2} \times(3+3+2)+\cdots \\
& +\frac{1}{9} \times \frac{1}{9} \times \frac{2}{9} \times(3+3+3)+\cdots \\
= & 6 \frac{3}{8} .
\end{aligned}
$$

applied only when the problem sizes are small. This is due to the fact that the formulas get involved with the enumeration of all possible partitions of a number of integers. The complexity of such enumeration is exponential. Computing by recursive formulas eases the problem to a certain extent. When the integers (parameters) becomes larger, it will be still extremely time-consuming to do the calculations. For instance, it takes $36.237 \mathrm{~s}$ for a program implemented on a Cyber $170 / 720$ system to calculate $N E 2(14,(14,3), 2,7,14)=$ 3.273526. Therefore, extensive experiments have been designed to test the approach of constructing perfect hash functions introduced in this paper.

Quite a number of strategies can be adopted for the partitioning of a given address into segments in the experiments. Here we introduce a strategy called geometric partition strategy. 


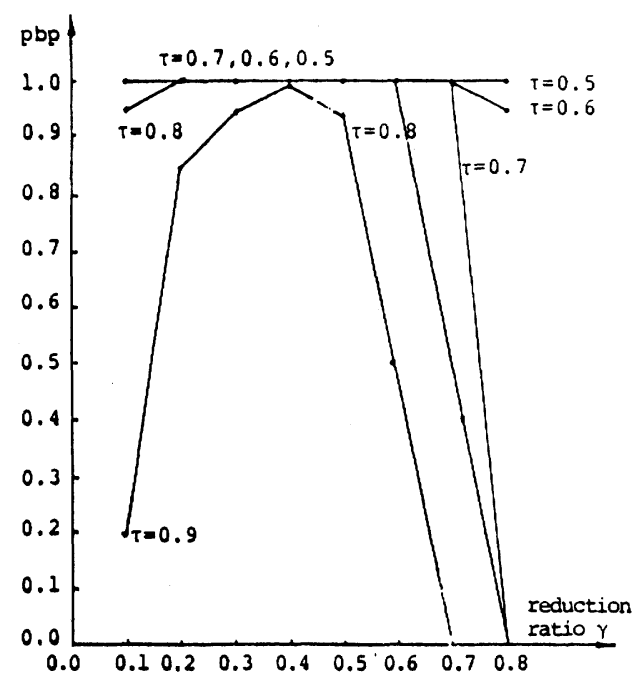

Fig. 8. Diagram of the pbp values of Experiment 1.

\section{Experiment 1-Explanation:}

1) Geometric partition strategy is tested.

2) Reduction ratio $\gamma$ varies from 0.1 to 0.8 in steps of 0.1 .

3) Loading factor $\tau$ varies from 0.5 to 0.9 in steps of 0.1 .

4) Number of random hash functions for each $h^{i}$ is 7 .

5) 100 independent sets with 100 nonequal keys in each are tested for each combination of $\gamma$ and $\tau$.

6) For each combination of $\gamma$ and $\tau$, we calculate the probability of being perfect pbp as the number of succeeded trials divided by the number of total trials (which is 100).

The results of this experiment are plotted in Fig. 8 .

From Fig. 8 we may observe immediately that we may have better chance to find a perfect hash function when the loading factor is smaller, which is quite natural. Also, all the curves corresponding to different $\tau$ values appear concave downward. It appears that there exists one and only one peak in each of these curves.

\section{Experiment 2-Explanation:}

1) Dynamic procedure (Procedure 4) for the construction of a perfect hash function is tested.

2) Number of keys $n$ varies from 40 to 50 , in steps of 1 , then from 50 to 500 in steps of 50 .

3) For each $n, 100$ independent sets with $n$ nonequal keys are tested.

4) The number of hash functions for constructing each $h^{i}$ is $1,3,7$, i.e., $s=1,3,7$.

The results of Experiment 2 are depicted in Figs. 9 and 10. Fig. 9 shows the average size of the address space assigned for each $(n, s)$ in the experiment. Note that there are 100 trials for each combination of $n$ and $s$. Fig. 10 shows the average number of times to apply $h_{j}^{i}$,s for calculating $H(K)$ for each combination of $n$ and $s$. For $n \leqslant 40$, these two figures, $L(n, s)$ and $N E D(n, s)$, are calculated through applying (A7) and (A9), respectively.

Example 6: Suppose that Procedure 4 will be used to construct a perfect hash function to map 300 nonequal keys into an address space. From Figs. 9 and 10, if 7 hash functions are used in constructing each $h^{i}$, the length of the address space will be about equal to 348 . The total size of the HIT table



Fig. 9. The average size of the address space assigned for each pair $(n, s)$ in Experiment 2.

created will be about $348 \times 3$ bits. Which is about 131 bytes. The expected number of times it is required to apply $h_{j}^{i}$ 's for calculating $H(K)$ will be about equal to 3.48 .

\section{Discussion}

This paper essentially proposed two procedures for constructing perfect hash functions. In the first procedure, the segmentation of the address space is preset. From Experiment 1 we can see that the way the address space is partitioned has great effect on the probability that a perfect hash function can be obtained. An interesting but unsolved problem is to show how to partition the address space so that we may maximize the probability of obtaining a perfect hash function by the construction procedure.

In the second procedure, the segmentation of the address space is obtained dynamically. If the address space is unlimited, the procedure can certainly construct a perfect hash function eventually. From Experiment 2, we observe that the ratio of the size of the address space constructed to the number of keys will approach a constant for each $s$ value, as the number of keys goes large. For example, when $s=7, L(n, s) \approx 7 / 6 n$. This provides us a very simple guide rule in applying Procedure 4. 


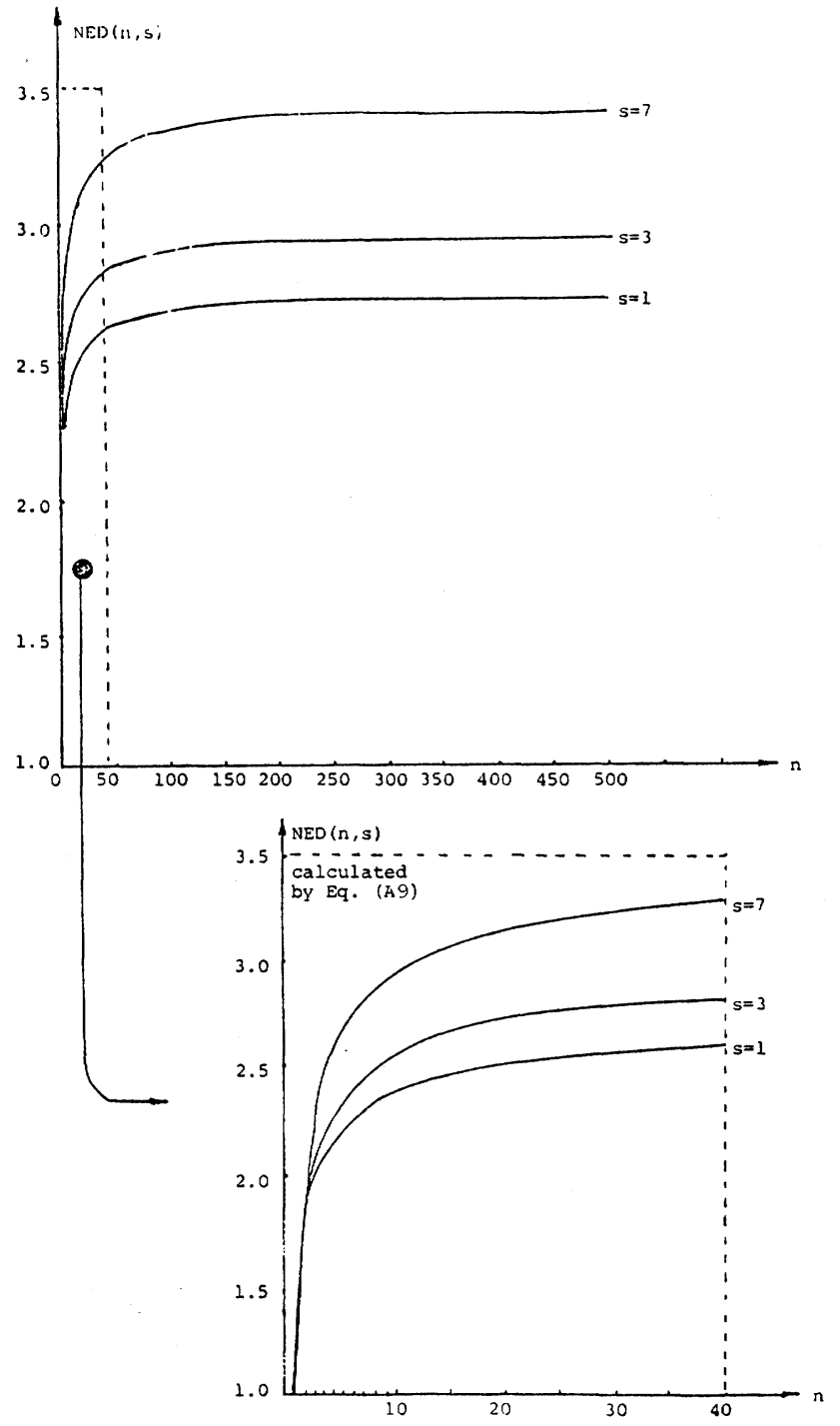

Fig. 10. Average number of times to apply $h_{j}^{i}$,s for $H(K)$ for each pair $(n, s)$ in Experiment 2.

\section{APPENDIX}

This Appendix lists formulas for calculating $Q_{k}^{s}, R_{k}^{s}, N E 1$, $N E 2, L(n, s)$, and $N E D(n, s)$ described in the context. $N E 1$, $N E 2$, and $N E D$ can be referred as the expected number of internal probes for finding the hash value of a key.

Suppose that $i$ singletons have been obtained by the successive application of $r$ random hash functions in the way described before. We define $\Delta P_{k}(n, m, i)$ as the probability of getting $k$ more singletons by applying the $(r+1)$ th random hash function.

Theorem A1: The probability

$$
\Delta P_{k}(n, m, i)=\left(\frac{i}{m}\right)^{n-i} \times \sum_{j=k}^{n-i} \frac{\left(\begin{array}{c}
n-i \\
j
\end{array}\right)}{i^{j}} e_{k}(j, m-i)
$$

where the function $e_{k}$ was defined in Theorem 1 .

Theorem $A 2$ : The probability distribution $Q_{k}^{s}(n, m)$ can be calculated by

$$
\begin{aligned}
Q_{k}^{s+1}(n, m) & =\sum_{j=0}^{k} Q_{k-j}^{s}(n, m) \Delta P_{j}(n, m, k-j) \\
& =\sum_{r=0}^{k} Q_{r}^{s}(n, m) \Delta P_{k-r}(n, m, r)
\end{aligned}
$$

with $Q_{k}^{1}(n, m)=P_{k}(n, m)$.

Theorem A3:

$$
\begin{array}{rl}
N E & 1(n, m, s+1, k) \\
= & \sum_{i_{s+1}=0}^{k}\left\{\left[\frac{k-i_{s+1}}{k} N E 1\left(n, m, s, k-i_{s+1}\right)\right.\right. \\
& \left.\left.+\frac{i_{s+1}}{k}(s+1)\right] \frac{Q_{k-i_{s+1}}^{s}(n, m) \cdot \Delta P_{i_{s+1}}\left(n, m, k-i_{s+1}\right)}{Q_{k}^{s+1}(n, m)}\right\}
\end{array}
$$

with

$$
N E 1(n, m, l, k)=1 \quad \text { for } 1 \leqslant k \leqslant n .
$$

Assuming that all $h_{j}^{i}$ 's are independent random hash functions, we have the following.

Theorem A4:

$$
\begin{aligned}
R_{k}^{s}(n, \overline{q m}, q)= & \sum_{k_{q=0}}^{k} \sum_{1}+k_{2}+\cdots+k_{q-1}=k-k_{q} \\
& \cdot \prod_{i=1}^{q} Q_{k_{i}}^{s}\left(n-\sum_{t=0}^{i-1} k_{t}, m_{i}\right) \\
= & \sum_{k_{q=0}}^{k} R_{k-k_{q}}^{s}(n, \overline{(q-1) m}, q-1) \\
& \cdot Q_{k_{q}}^{s}\left(n-\left(k-k_{q}\right), m_{q}\right)
\end{aligned}
$$

with $R_{k}^{s}(n, \overline{1 m}, 1)=Q_{k}^{s}\left(n, m_{1}\right)$.

Theorem A5:

$N E 2(n, \overline{q m}, q, s, k)$

$$
\begin{aligned}
= & \left\{\sum_{q=0}^{k}\left(\frac{k-l_{q}}{k}\right) N E 2\left(n, \overline{(q-1) m}, q-1, s, k-l_{q}\right)\right. \\
+ & \left.\left.\frac{l_{q}}{k}\left[(q-1) s+N E 1 \overline{\left(n-\left(k-l_{q}\right)\right.}, m_{q}, s, l_{q}\right)\right]\right\} \\
& \frac{R_{k-l q}^{s}\left(n,(\overline{q-1) m}, q-1) \cdot Q_{l q}^{s}(n-(k-l q), m q)\right.}{R_{k}^{s}(n, \bar{m}, q)}
\end{aligned}
$$

with $N E 2(n, \overline{1 m}, 1, s, k)=N E 1\left(n, m_{1}, s, k\right)$ for all $0 \leqslant k \leqslant n$.

Theorem A6:

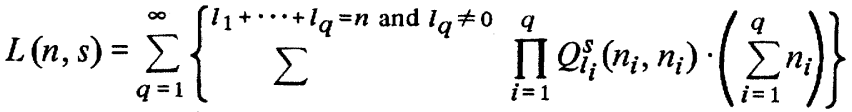

when

$$
n_{i}=n-\sum_{t=0}^{i-1} l_{t}, l_{0}=0 .
$$

Theorem A7:

$$
L(n, s)=\frac{1}{1-Q_{0}^{s}(n, n)}\left[n+\sum_{k=1}^{n-1} L(k, s) Q_{n-k}^{s}(n, n)\right]
$$

with $L(0, s)=0, L(1, s)=1$.

Theorem A8:

$$
\begin{aligned}
& N E D(n, s)=\sum_{q=1}^{\infty}\left\{\begin{array}{c}
l_{1}+\cdots+l_{q}=n \text { and } l_{q} \neq 0 \\
\sum^{2}
\end{array}\right. \\
& \left.\cdot \prod_{i=1}^{q} Q_{l_{i}}^{s}\left(n_{i}, n_{i}\right) N E 2(n, \bar{m}, q, s, n)\right\}
\end{aligned}
$$


where

$$
n_{i}=n-\sum_{t=0}^{i-1} l_{t}, l_{0}=0, \quad \text { and } \bar{m}=\left(n_{1}, n_{2}, \cdots, n_{q}\right)
$$

Theorem A9:

$$
\begin{aligned}
N E D(n, s)= & \frac{1}{1-Q_{0}^{s}(n, n)} \\
& \cdot s \cdot Q_{0}^{s}(n, n)+N E 1(n, n, s, n) Q_{n}^{s}(n, n) \\
& +\sum_{k=1}^{n-1}\left[\left(\frac{k}{n}\right) N E 1(n, n, s, k)\right. \\
& \left.\left.+\left(\frac{n-k}{n}\right)(s+N E D(n-k, s))\right] \cdot Q_{k}^{s}(n, n)\right\}
\end{aligned}
$$

\section{REFERENCES}

[1] M. R. Anderson and M. G. Anderson, "Comments on perfect hashing functions: A single probe retrieving method for static sets," Commun. Ass. Comput. Mach., vol. 22, p. 104, Feb. 1979.

[2] W. Buchholz, "File organization and addressing," IBM Syst. J., vol. 2, pp. 86-111, June 1963.

[3] R. J. Cichelli, "Minimal perfect hash functions made simple," Commun. Ass. Comput. Mach., vol. 23, pp. 17-19, Jan. 1980.

[4] F. N. Davis and D. E. Barton, Combinational Chances. London: Griffin, 1962

[5] M. W. Du, K. F. Jea, and D. W. Shieh, "The study of new perfect hash schemes," in Proc. COMPSAC'80, Chicago, IL, Oct. 1980, pp. 341-347.

[6] M. W. Du and H. C. Lin, "The design of a memory saving Chinese input/output system," in Proc. NCS, Taipei, Dec. 1979, pp. 8.18.10 .

[7] W. Feller, An Introduction to Probability Theory and Its Applications, vol. 1. New York: Wiley, 1950.

[8] S. P. Ghosh, Data Base Organization for Data Management. New York: Academic, 1977.

[9] G. Jaeschke and G. Osterburg, "On Chichelli's minimal perfect hash functions method," Commun. Ass. Comput. Mach., vol. 23, pp. 728-729, Dec. 1980 .

[10] G. Jaeschke, "Reciprocal hashing: A method for generating minimal perfect hashing functions," Commun. Ass. Comput. Mach., vol. 24 , pp. $829-833$, Dec. 1981

[11] K. F. Jea, "The study of the static properties of a new perfect hash scheme," Master thesis, Nat. Chiao Tung Univ., Hsinchu, Taiwan, R.O.C., May 1980.

[12] L. R. Johnson, "An indirect chaining method for addressing on secondary keys," Commun. Ass. Comput. Mach., vol. 4, pp. 218222, May 1981.

[13] D. E. Knuth, The Art of Computer Programming, Vol. 3, Sorting and Searching. Reading, MA: Addison-Wesley, 1973.

[14] C. L. Liu, Introduction to Combinatorial Mathematic. New York: McGraw-Hill, 1968

[15] V. Y. Lum, P.S.T. Yuen, and M. Dodd, "Key-to-address transform techniques: A fundamental performance study on large existing formatted files," Commun. Ass. Comput. Mach., vol. 14, pp. 228-239, Apr. 1971.

[16] W. D. Maurer and T. G. Lewis, "Hash table method," Comput. Surveys, vol. 7, pp. 5-19, Mar. 1975.

[17] R. Morris, "Scatter storage techniques," Commun. Ass. Comput. Mach., vol. 11, pp. 38-44, Jan. 1968.

[18] C. A. Olson, "Random access file organization for indirectly accessed records," in Proc. ACM 24th Nat. Conf., 1969, pp. 539-549.

[19] W. W. Peterson, "Addressing for random-access storage," IBM J. Res. Develop., vol. 1, pp. 130-146, Apr. 1957.

[20] G. Schay and W. G. Spruth, "Analysis of a file addressing method," Commun. Ass. Comput. Mach., vol. 5, pp. 459-462, Aug. 1962.

[21] D. G. Severance, "Identifier search mechanisms: A survey and generalized model," Comput. Surveys, vol. 6, pp. 175-194, Sept. 1974.

[22] R. Sprugnoli, "Perfect hashing functions: A single probe retrieving method for static sets," Commun. Ass. Comput. Mach., vol. 20, pp. 841-850, Nov. 1977.

[23] M. Tainiter, "Addressing for random-access storage with multiple bucket capacities," J. Ass. Comput. Mach., vol. 10, pp. 307-315, July 1963.

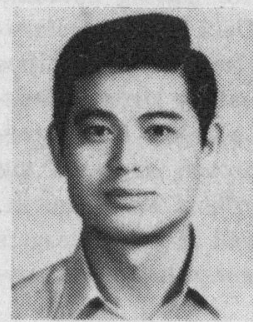

M. W. Du (S'70-M'72) received the B.S.E.E. degree from the National Taiwan University in 1966 and the Ph.D. degree from The Johns Hopkins University, Baltimore, MD, in 1972.

He is currently the Director of the Institute of Computer Engineering, National Chiao Tung University, Hsinchu, Taiwan. His research interests include fault diagnosis, automata theory, algorithm design and analysis, database design, and Chinese $\mathrm{I} / \mathrm{O}$ design.

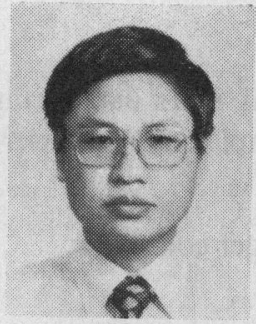

T. M. Hsieh was born in Taiwan, on November 15,1947 . He received the B.S. degree in electrical engineering from the Chung Yuan University, Chung-Li, Taiwan, in 1970 and the M.S. degree in electrical engineering from the Institute of Electronics, National Chiao Tung University, Hsinchu, Taiwan, in 1974.

He is now working towards the Ph.D. degree in the Institute of Electronics, National Chiao Tung University. He previously worked for Telecommunication Laboratories, Chung-Li, Taiwan, before joining the Department of Electronic Engineering at the Chung Yuan University in 1975, where he is currently an Associate Professor. His research interests include database systems, faulttolerant computing, microprocessor/microcomputer based systems.

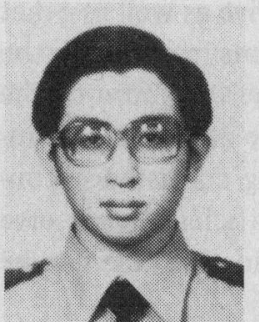

K. F. Jea was born in Taiwan on May 13, 1956 He received the B.S. and M.S. degrees in computer science from the National Chiao Tung University, Hsinchu, Taiwan, in 1978 and 1980 , respectively.

From 1977 to 1980 , he served as a Research Assistant in the Department of Computer Science, National Chiao Tung University. He is currently working towards the Ph.D. degree in computer science at the University of Wisconsin, Madison. His research interests include database design, programming language and compiler design, algorithm design, and analysis.

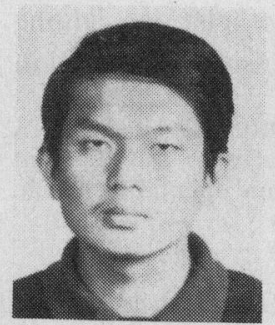

D. W. Shieh was born in Taiwan, Republic of China, on January 1,1952 . He received the B.S. and M.S. degrees in computer science from the National Tung University, Taiwan, Republic of China, in 1976 and 1980 , respectively.

Currently, he is a System Engineer at the System Development Center, Institute for Information Industry, Taiwan, Republic of China. His primary job is software system development. 